\title{
Las representaciones sociales del cuidado infantil desde una perspectiva de género. Principales resultados de la Encuesta Nacional sobre Representaciones Sociales del Cuidado
}

\author{
Karina Batthyány \\ Natalia Genta \\ Valentina Perrotta
}

Universidad de la República. Facultad de Ciencias Sociales. Departamento de Sociología

karina.batthyany@cienciassociales.edu.uy

natalia.genta@gmail.com

vperrottag@gmail.com

Recibido: 04-11-2013

Aceptado: 12-03-2014

\section{Resumen}

El presente artículo tiene como objetivos presentar y analizar resultados de investigación sobre representaciones sociales del cuidado infantil. A partir de una encuesta realizada a nivel nacional, se analizan específicamente las situaciones deseables sobre esta cuestión, las obligaciones percibidas de madres y padres y la posible articulación entre trabajo remunerado y cuidado. Asimismo, el artículo aborda las contribuciones de dichos resultados a la política pública de cuidados en Uruguay. Los resultados muestran la fuerte presencia del familismo en las representaciones, puesto que es la situación más deseable para el cuidado de los niños pequeños. Se constató la fuerza del mandato de género en el rol cuidador de la identidad femenina, que trasciende las distintas posiciones sociales de las mujeres. Ellas están más dispuestas que los varones a abandonar o flexibilizar sus empleos para dedicarse al cuidado de niños.

Palabras clave: cuidados; cuidado de niños; cuidados familiares; imaginarios; familismo; mentalidades; rol familiar.

Abstract. Social representations of child care from a gender perspective: Main results of the National Survey on Social Care Representations

This article aims to present and discuss research results on social representations of child care. Using a national survey, we examine desirable child care situations, the perceived obligations of parents, and the possible link between paid work and care. In addition, the article discusses the contributions of the survey results to public policy 
on care in Uruguay. The results show the powerful presence of "familism" in representations. The most desirable situation in early childhood is care provided at home, especially by family members. We found that the gender mandate of caregiving has a strong influence on female identity, which transcends women's social positions. Women are more likely than men to leave their jobs or seek flexible working arrangements to provide child care.

Keywords: childcare; family care; imaginary; familism; mentalities; family role.

\section{Sumario}
1. Introducción
4. Principales resultados
2. Nociones de partida sobre los cuidados
5. Principales conclusiones
3. Apartado metodológico
Referencias bibliográficas

\section{Introducción}

En el año 2010, Uruguay da inicio a un proceso de construcción de un sistema nacional de cuidados que busca transformar la matriz familista ${ }^{1}$ de distribución de cuidados, hacia una de corresponsabilidad entre Estado, familias y mercado, así como entre mujeres y varones (Consejo Nacional de Política Social, 2012: 12).

En el marco de este proceso, el proyecto de investigación que da origen a este artículo se propone investigar un vacío de conocimiento identificado con anterioridad (Aguirre, 2009): las representaciones sociales de la población en relación con el cuidado. Dicho proyecto, llamado Hacia un sistema nacional de cuidados: Representaciones sociales de la población y propuestas para el cuidado de los dependientes, formó parte de la convocatoria de proyectos de alto impacto social, promovida por la Agencia Nacional de Investigación e Innovación (ANII), y fue realizado por el Grupo de Investigación sobre Sociología de Género del Departamento de Sociología de la Facultad de Ciencias Sociales (Universidad de la República) ${ }^{2}$. El mismo se propuso como objetivo general avanzar en el conocimiento y la comprensión del cuidado como elemento constitutivo del bienestar social desde una perspectiva de

1. Los términos familismo o familista, utilizados indistintamente en este artículo, caracteriza a aquellas representaciones sociales del cuidado que señalan como más deseable que éste sea asumido por personas de la familia. De la misma forma, se utiliza para identificar a la organización social del cuidado donde la responsabilidad del cuidado es asumida mayormente o en exclusividad por las familias. Dicha acepción está basada en la categorización de los regímenes de bienestar mencionada en el marco conceptual.

2. Este proyecto contó con el apoyo adicional del Fondo de Población de Naciones Unidas (UNFPA), de la Entidad de las Naciones Unidas para la Igualdad de Género y el Empoderamiento de las Mujeres y de la División de Asuntos de Género de la CEPAL. Esta propuesta se desarrolló de manera articulada con el Instituto Nacional de las Mujeres (INMUJERES), del Ministerio de Desarrollo Social (MIDES), como organismo rector de las políticas de equidad de género en Uruguay. 
género y derechos, a fin de facilitar la implementación de un sistema nacional de cuidados en Uruguay.

Este artículo tiene como objetivo presentar los principales resultados de dicha investigación, colocando el foco en las representaciones sociales del cuidado infantil. El artículo responde a las preguntas siguientes: ¿cuáles son las representaciones sociales de cuidado de la población uruguaya en relación con el cuidado infantil?, ¿cuáles son las contribuciones principales surgidas del estudio de las representaciones del cuidado infantil a la política pública de cuidados en el país?

Los objetivos específicos de este artículo son:

1. Presentar y analizar los resultados de investigación sobre: $a$ ) las situaciones deseables del cuidado infantil; $b$ ) las percepciones sobre obligaciones de madres y padres y $c$ ) las decisiones que tomarían varones y mujeres en el mercado laboral frente a la imposibilidad de conseguir licencias para el cuidado infantil.

2. Analizar la contribución del estudio de estas representaciones para la política pública de cuidados en el país.

Las hipótesis de investigación referidas a las preguntas específicas de este artículo establecían que las situaciones deseables del cuidado infantil, las obligaciones de madres y padres, así como las decisiones que tomarían varones y mujeres en el mercado laboral frente a la imposibilidad de conseguir licencias para el cuidado infantil estarían mediadas por el nivel socioeconómico, el sexo y el contacto con situaciones de cuidado. Se partía del hecho de que el nivel socioeconómico estaba relacionado con las posibilidades de acceso a servicios de atención infantil, lo cual determinaba la experiencia con distintas modalidades de cuidado extrafamiliar y, de esta forma, incidía en la percepción sobre lo deseable. La variable sexo determinaría estas representaciones, debido a que los mandatos de género establecen obligaciones diferenciales en relación con el cuidado de niños pequeños. Por último, el contacto con situaciones de asistencia incidiría en las representaciones, debido a la experiencia vivida de enfrentarse a la necesidad de cuidado.

El presente artículo se organiza de la siguiente manera. El primer apartado está destinado a la presentación de algunas discusiones teóricas que son claves para la interpretación de los resultados desde una perspectiva de género. Luego, se presenta el apartado metodológico con las características de la estrategia de investigación utilizada en el estudio que da origen a este artículo. A continuación, se exponen los hallazgos en las siguientes dimensiones analizadas: situación deseable de cuidado infantil; edad ideal para la entrada en un centro infantil o para estar al cuidado contratado en domicilio; representaciones sobre el «deber ser» de padres y madres en el cuidado, y decisiones que tomarían varones y mujeres en el mercado laboral frente a la imposibilidad de conseguir licencias para el cuidado infantil. Finalmente, se presentan las principales conclusiones, en las que se vinculan los hallazgos de investigación con la política pública de cuidados. 


\section{Nociones de partida sobre los cuidados}

La noción de cuidados se ha vuelto clave para la investigación y el análisis de las políticas de protección social con perspectiva de género. Se trata de un concepto que, lejos de suscitar consenso, presenta diversas definiciones. Los debates académicos sobre su contenido se remontan a los años setenta en los países anglosajones y fueron impulsados por las corrientes feministas en el campo de las ciencias sociales ${ }^{3}$.

Partiendo y reconociendo una noción amplia de cuidado ${ }^{4}$, se parte de aquélla que resulta más aplicable al análisis de las políticas públicas. De esta forma, se conceptualiza al cuidado infantil como la acción de ayudar a un niño a desarrollarse y a facilitarle bienestar en su vida cotidiana. Engloba, por tanto, hacerse cargo del cuidado material que implica un «trabajo», del cuidado económico que implica un "costo económico" y del cuidado psicológico que implica el desarrollo de un vínculo afectivo, emotivo, sentimental. Ello puede ser realizado de manera honoraria o benéfica por parientes en el marco de la familia, o de manera remunerada en el marco o no de la familia. La naturaleza de la actividad variará según se realice o no dentro de la familia y también de acuerdo con el hecho de si se trata o no de una tarea remunerada 5 (Aguirre, 2003b; Batthyány, 2002). Los cuidados no son, por definición, en sí mismos, de carácter remunerado o no remunerado, sino que ello resulta como consecuencia de elecciones políticas, valoraciones culturales compartidas y sistemas de género.

El trabajo de cuidado está marcado por la relación de servicio y de preocupación por los otros (Hochschild, 2003), además, se realiza cara a cara entre dos personas y genera lazos de proximidad, en una situación de dependencia, pues una es tributaria de la otra para su bienestar y mantenimiento.

En definitiva, la definición de cuidado implica integrar los aspectos vinculares junto a los aspectos materiales. Dentro de la familia, estas tareas involucran simultaneidad de papeles, roles y responsabilidades, que, para ser captados, requieren considerar conceptos como los de dirección y gestión, no fácilmente traducibles en estimaciones de tiempo, intensidad o esfuerzo (Durán, 2003).

La especificidad del trabajo de cuidado — se dé dentro o fuera de la familia - es la de estar basado en lo relacional (Pérez Orozco, 2006). En el marco de la familia, su carácter a la vez obligatorio y desinteresado le otorga una dimensión moral y emocional —ya que involucra las emociones que se expresan en el seno familiar, al mismo tiempo que contribuye a construirlas y man-

3. Una buena síntesis del estado actual del debate acerca de la noción de cuidados se encuentra en C. Thomas (20II), «Deconstruyendo los conceptos de cuidados», en: Carrasco, BorDERÍAs y Torns (eds.). El trabajo de cuidados: Historia, teoría y políticas. Madrid: Catarata.

4. Nos referimos a la definición propuesta por Fisher y Tronto (1990): «El cuidado es una actividad específica que incluye todo lo que hacemos para mantener, continuar y reparar nuestro mundo, de manera que podamos vivir en él tan bien como sea posible. Ese mundo incluye nuestros cuerpos, nuestro ser y nuestro ambiente, todo lo que buscamos para entretejer una compleja red del sostenimiento de la vida» (Fisher y Tronto, 1990, en Montaño, 2010: 27).

5. Esta definición fue inspirada por Letablier (2001). 
tenerlas- Se trata de una tarea esencialmente realizada por mujeres, tanto si es llevada a cabo por parte de parientes como si se exterioriza a través de la contratación de servicios.

Actualmente, las mujeres en Uruguay cuentan con mayor autonomía económica respecto a décadas anteriores, producto del aumento generalizado de la tasa de actividad femenina, que pasó del 40,4\% en 1986 al 55,6\% en $2013^{6}$. Sin embargo, las mujeres enfrentan grandes problemas para articular los tiempos de trabajo remunerado y los tiempos de los cuidados, debido al desbalance en la dedicación de madres y padres y a la insuficiencia de políticas que atiendan a las necesidades de las personas dependientes. $\mathrm{Al}$ respecto, un elemento bastante útil que se ha desarrollado en la región en los últimos años son las encuestas sobre uso del tiempo (EUT), que nos permiten aproximarnos empíricamente a la división sexual del trabajo dentro de los hogares y observar cambios y permanencias (Batthyány, 2010). La Encuesta sobre Uso del Tiempo y Trabajo no Remunerado, realizada en Montevideo y su área metropolitana en 2003, mostraba que las madres dedican al cuidado de sus hijos menores de 12 años el doble de horas que los padres (Aguirre y Batthyány, 2005), tendencia corroborada en la Encuesta Nacional de Uso del Tiempo realizada en Uruguay en 2007 (Batthyány, 2009).

Esta desigual dedicación tiene consecuencias de género relevantes, pues, cuando las mujeres de las familias son las principales proveedoras del bienestar, éstas deben o bien excluirse del mercado laboral o bien enfrentarse a mayores dificultades que sus pares masculinos para articular trabajo productivo $y$ reproductivo.

Dichas consecuencias fueron evidenciadas en varias investigaciones, donde se mostraba que las trabajadoras que son madres desarrollan estrategias complejas de atención familiar para incorporarse al mercado laboral, debido a las dificultades de acceso al cuidado de carácter institucional o familiar (Batthyány, 2002; Aguirre, 2003a; Batthyány, 2004). Las investigaciones evidenciaron conductas discriminatorias a las que se enfrentaban las mujeres madres para el ejercicio de sus derechos en los ámbitos de trabajo, así como mecanismos por los cuales se establecía una distancia entre el reconocimiento formal de estos derechos y su ejercicio efectivo (Batthyány, 2002; Aguirre, 2003a; Batthyány, 2004). Por otro lado, al analizar la cobertura de atención infantil en Uruguay, se concluía que no se concebían los cuidados infantiles para niños pequeños (0 a 3 años) como un derecho de los padres y los niños ni como una responsabilidad por parte del Estado, de modo que las mayores dificultades de acceso se presentaban para estas edades (Batthyány, 2001).

Si bien, históricamente, la dedicación a las personas se ha resuelto en el seno de las familias, en nuestros días, han cambiado tanto las necesidades como quienes pueden prestar esos cuidados. Actualmente, asistimos a lo que se denomina "crisis del cuidado". Se trata de un momento histórico en que existe «un déficit» por el desajuste entre la demanda — necesidades de atención-y la

6. Datos extraídos de la pagina del Instituto Nacional de Estadística (www.ine.gub.uy). 
oferta disponible. El aumento sostenido desde la década de 1970 de la incorporación femenina al mercado de empleo, junto con una mayor búsqueda de autonomía, reducen el número de mujeres disponibles en forma exclusiva para estas tareas. En forma paralela, se han producido ciertas transformaciones familiares caracterizadas por la reducción de la proporción de hogares biparentales con hijos en los que el varón es el único sostén económico del hogar y la mujer es ama de casa a tiempo completo. Esto también contribuye a que haya menos personas en condiciones de brindar asistencia (Arriagada, 2007).

En los últimos años, el debate en torno al tema de los cuidados se complejizó y se desplazó de la esfera estrictamente privada de la familia, para pasar a la esfera pública de los estados de bienestar. Se cuestiona el rol de la Administración en el apoyo a las familias, al tiempo que se reformula la cuestión en términos del modo en que se comparten las responsabilidades de cuidado entre diversas instituciones: el Estado, las familias, el mercado y las organizaciones comunitarias.

\subsection{La distribución social de los cuidados}

El foco en la noción de cuidados y en la distribución inequitativa de los mismos implica profundizar en el debate conceptual acerca de los regímenes de bienestar. Autoras como Daly y Lewis plantean que el cuidado se ha constituido como categoría central para comprender la forma y la naturaleza de los estados de bienestar contemporáneos (Daly y Lewis, 2011: 226).

Luego de sostenidas críticas feministas a sus modelos de "estado mercado", Esping Andersen se propone revisar sus análisis de los regímenes de bienestar, incorporándoles dos esferas: las familias y la comunidad, reconociendo el importante aporte de estos actores en la provisión de bienestar. Por tanto, siguiendo a Esping Andersen (2000), el bienestar es provisto mediante la combinación de la acción de cuatro actores: la comunidad, el mercado, las familias y el Estado. A partir de estas categorías, se han analizado los regímenes en distintos países de la región, tomando en cuenta no solo los actores involucrados en la organización de los cuidados (Estado, mercado, comunidad y familias), sino también las funciones que cumplen en términos de fuentes de bienes, servicios, tiempo y dinero (Aguirre, 2008; Martínez, 2009).

La distribución en la provisión de bienestar entre estos actores determinará el ejercicio de derechos de las mujeres y, por tanto, la posibilidad de desarrollar una ciudadanía plena. En función de esta distribución de responsabilidades, Aguirre clasifica a los regímenes de bienestar según el peso que tengan los servicios del Estado, la extensión de los servicios del mercado y la participación de las familias y las redes informales en ellos. De esta forma, siguiendo a Sarraceno (1994) y Sainsbury (2000), los identifica como familistas o desfamiliarizadores. En el régimen familista típico, la responsabilidad principal del bienestar corresponde a las familias y a las mujeres en las redes de parentesco. Por su parte, en el régimen desfamiliarizador, hay una derivación de las funciones de bienestar hacia las instituciones públicas y hacia el mercado. La autora identifica otro 
escenario posible para la equidad social y de género en el que se desarrollen políticas de corresponsabilidad entre familias, Estado y mercado, de forma tal que se favorezca la ampliación del ejercicio de derechos sociales, económicos y políticos de las mujeres.

Por su parte, Martínez (2009) afirma que, en los estados de bienestar latinoamericanos, la desfamilizarización es baja, predomina la división sexual del trabajo y el trabajo no remunerado tiene un papel central para el cuidado de personas dependientes. Martínez y Voorend (2013) señalan que, en América Latina, los regímenes de bienestar no son tan determinantes de las desigualdades originadas en los mercados laborales como de las originadas en la políticas sociales y en los cuidados que se realizan de manera no remunerada por parte de las mujeres en el interior de las familias.

Recientemente, se ha enfatizado en la dimensión de los cuidados como derecho modificando el concepto de ciudadanía. El derecho al cuidado implica considerarlo desde su doble circunstancia de personas que precisan cuidados y que cuidan. Ello conlleva que el Estado garantice el derecho de recibir atención en distintas circunstancias y momentos del ciclo vital, lo cual evita que la satisfacción de esa necesidad se determine por la lógica de mercado, la disponibilidad de ingresos o la presencia de redes vinculares. También implica garantizar el derecho a elegir si se desea o no cuidar, así como condiciones laborales dignas, a fin de valorizar la tarea, tanto desde el punto de vista social como económico. Esto implica una nueva concepción de la relación entre individuo, familia y Estado basada en la responsabilidad compartida de los cuidados de las personas (Pautassi, 2010).

Desde el enfoque de derechos, se cuestiona el papel del Estado como subsidiario, destinado a compensar las prestaciones que no se obtienen en el mercado, y se favorece el papel del Estado como garante de derechos. Por tanto, los tres pilares clásicos del bienestar vinculados a la salud, la educación y la seguridad social están siendo complementados con el denominado cuarto pilar, que reconoce el derecho a recibir atención en situaciones de dependencia (Navarro, 2005).

\subsection{La importancia de las representaciones sociales del cuidado}

El proyecto que da origen a este artículo se propone investigar un vacío de conocimiento identificado con anterioridad (Aguirre, 2009): las representaciones sociales de la población en relación con el cuidado. Conocer las representaciones sociales permite orientar la formulación de políticas de cuidados a través de la identificación de las barreras culturales para la aceptación de distintos servicios e intervenciones estatales. Asimismo, permite elaborar una línea de base previa a la puesta en marcha de la política pública de cuidados que podrá volver a medirse luego de su implementación.

Repasando el concepto de representación social, recordemos que Moscovici lo propuso en 1961. Desde entonces, el desarrollo de la teoría sobre las representaciones sociales ha permeado las ciencias sociales, porque constituye 
un enfoque que unifica e integra lo individual y lo colectivo, lo simbólico y lo social, el pensamiento y la acción.

Las representaciones sociales dan cuenta de un conocimiento práctico productor y constructor de una realidad social compartida por un colectivo y a través de las cuales se intenta dominar ese entorno, comprenderlo y explicarlo.

La teoría de las representaciones sociales postulada por Moscovici (1979) constituye un marco interesante para una aproximación compleja al universo de significados y valores que portan los individuos sobre la temática del cuidado. Araya Umaña propone que las representaciones sociales constituyen sistemas cognitivos en los que es posible reconocer la presencia de estereotipos, opiniones, creencias, valores y normas que orientan las prácticas. Se constituyen como fuerza normativa que instituye los límites y las posibilidades de acción de varones y mujeres (Araya Umaña, 2002).

La importancia del estudio de las representaciones sociales para los estudios de género radica en hacer visibles las creencias, los valores, los supuestos ideológicos que construyen, con base en las diferencias biológicas, las desigualdades sociales entre mujeres y hombres. El conocimiento de estos elementos es clave para su transformación y su consideración en las políticas públicas que promueven la equidad de género.

\section{Apartado metodológico}

La estrategia metodológica de la investigación que da origen a este artículo se orientó a caracterizar las distintas representaciones sobre el cuidado y su relación con variables como nivel socioeconómico, edad, nivel educativo, contacto con situaciones de cuidado y convivencia con niños pequeños.

Las principales dimensiones abordadas en dicha investigación fueron las siguientes: a) necesidades y demandas de cuidado; $b$ ) situaciones más deseables para el cuidado infantil y de adultos mayores; $c$ ) percepciones sobre responsabilidades de los diferentes actores en el cuidado; $d$ ) percepciones sobre obligaciones familiares y de varones y mujeres en el cuidado; $e$ ) posibilidades que ofrece el mercado laboral para brindar cuidado; $f$ ) decisiones respecto a la situación laboral ante las necesidades de atención, y $g$ ) recursos disponibles para el cuidado infantil.

Se realizó una encuesta "cara a cara» de la población uruguaya sobre la base de una muestra representativa estratificada por ingresos y por región de 800 casos. Se diseñó una muestra por conglomerado y polietápica, con aplicación de cuotas por sexo y edad en el hogar para la selección de la persona encuestada. El universo de la encuesta fueron varones y mujeres mayores de 18 años residentes en conjuntos urbanos de más de 5.000 habitantes.

El formulario de encuesta contó con cinco bloques diferenciados. El primero indagó en la existencia de contacto y dedicación horaria al cuidado niños y niñas de 0 a 3 años y de 4 a 12 años. Asimismo, obtuvo datos sobre necesidades de atención debidas a dificultades físicas y sobre la existencia de cobertura de esas necesidades. El segundo bloque relevó información sobre las soluciones y las situa- 
ciones más deseables de cuidado. El tercer bloque indagó sobre las obligaciones y las expectativas percibidas sobre el cuidado, las dificultades para cuidar, el deber ser del cuidado y el significado del tiempo dedicado al mismo. El cuarto bloque, exclusivo para trabajadores remunerados, obtuvo datos sobre flexibilidad laboral para el cuidado y sobre las decisiones frente a la imposibilidad de articular trabajo remunerado y cuidados. El quinto bloque estuvo destinado a relevar tres tipos de información sobre los gastos que las personas realizarían para costear servicios de cuidado infantil de calidad, quiénes eran los referentes para la crianza de los niños y la distribución percibida de responsabilidades de atenciones a niños de distintas edades y a adultos mayores, entre Estado, mercado, familias y comunidad.

En los dos últimos bloques, se obtuvo información sociodemográfica sobre las personas encuestadas, la composición del hogar y su nivel socioeconómico. Para la construcción de éste último, se utilizó el índice de nivel socioeconómico para estudios de mercado y opinión pública (INSE), validado por la Facultad de Ciencias Sociales (2006), que permite clasificar al hogar en siete estratos en función de sus dimensiones educativas, ocupacionales y de acceso, así como de su disponibilidad de recursos económicos.

A diferencia de gran parte de las investigaciones sobre representaciones sociales que utilizan diseños de tipo cualitativo, por medio de los cuales no es posible generalizar resultados, la metodología utilizada en este estudio, a partir de una encuesta representativa, permitió que los resultados fueran generalizables a la población del país. La representatividad de los datos a nivel nacional es una de las grandes fortalezas de la investigación, porque otorga legitimidad a los resultados para ser considerados en el diseño de políticas de cuidado actualmente en construcción.

En segundo lugar, la metodología utilizada posibilitó el estudio de las articulaciones entre representaciones y variables estructurales (nivel socioeconómico (NSE), nivel educativo, sexo, edad y composición familiar). Esta encuesta permitió identificar las diferencias en las representaciones sociales del cuidado a partir de las distintas posiciones que las personas ocupan en la estructura social. Esta información habilita la generación de políticas de cuidados diferenciadas.

\section{Principales resultados}

Respondiendo a los objetivos ya mencionados del artículo, a continuación, se presenta el análisis de los resultados de la investigación en función de las siguientes dimensiones: $a$ ) situaciones deseables del cuidado infantil; $b$ ) percepciones sobre obligaciones de madres y padres en el cuidado infantil, y c) decisiones respecto a la situación laboral ante las necesidades de cuidado.

4.1. Familias, centros infantiles, personas contratadas... ¿Cuál es la situación más deseable para el cuidado infantil?

Comenzaremos por presentar cuál es la situación más deseable para el cuidado infantil durante la jornada laboral para la población uruguaya. Encontramos 
Tabla 1. Situación más deseable para el cuidado de niños menores de 2 años durante la jornada laboral (porcentaje de la población total dividida por sexos)

\begin{tabular}{llrrr}
\hline & & Varones & Mujeres & \multicolumn{1}{c}{ Total } \\
\hline Cuidado domiciliario & Que le cuidara todo el tiempo entre usted & 40,2 & 27,3 & 33,4 \\
& $\begin{array}{l}\text { y su pareja } \\
\text { Que le cuidara principalmente usted }\end{array}$ & 3,5 & 13,5 & 8,8 \\
& $\begin{array}{l}\text { Que le cuidara principalmente su pareja } \\
\text { Que le cuidara entre su pareja, usted y un }\end{array}$ & 9,6 & 0,5 & 4,8 \\
& $\begin{array}{l}\text { familiar cercano (distintas combinaciones } \\
\text { de dedicación entre parientes) }\end{array}$ & 20,9 & 18,3 \\
& $\begin{array}{l}\text { Que le cuide una persona contratada y } \\
\text { Otras opciones con persona contratada }\end{array}$ & 10,1 & 10,5 & 10,3 \\
& Subtotal referente al cuidado domiciliario & 78,7 & 72,7 & 75,5 \\
\hline Cuidado institucional & $\begin{array}{l}\text { Que asistiera a un centro infantil y otras } \\
\text { opciones combinadas con centro infantil }\end{array}$ & 18,9 & 25,2 & 22,2 \\
& (cuidado familiar y en centro infantil) & & & \\
& No sabe / No contesta & 2,4 & 2,1 & 2,3 \\
\hline Total & & 100 & 100 & 100 \\
\hline
\end{tabular}

Fuente: Encuesta Nacional sobre Representaciones Sociales del Cuidado.

que, para el 75,5\% de personas encuestadas, el cuidado domiciliario es la opción más deseable y, dentro de esta modalidad, prácticamente dos tercios de la población (un 65,3\%) se inclina a favor de que el cuidado se realice sólo por medio de la pareja (ambos o alguno de los dos) y algún familiar cercano. Es muy importante considerar este dato en el momento de diseñar políticas de cuidado, para que éstas incluyan mecanismos que habiliten a las familias a llevar adelante dichas expectativas, dado que, actualmente, se presentan múltiples dificultades para el cuidado familiar de los niños menores de 2 años y ello convierte a esta expectativa en una realidad inviable para gran parte de la población.

Dentro de las opciones de cuidado familiar, el 40,2\% de los varones cree que la situación más deseable para la atención de menores de 2 años durante la jornada laboral es que el mismo sea compartido con la pareja, lo que resulta un dato alentador, dado que estos varones parecen reconocer que la responsabilidad de los hijos no es exclusiva de las mujeres. Sin embargo, hallamos una contradicción en este punto entre lo que los varones señalan como la situación más deseable para el cuidado infantil y las prácticas que surgen de las encuestas sobre uso del tiempo realizadas en el país (Aguirre y Batthyány, 2005; INE, 2008), que evidencian la mayor participación de las mujeres en estas tareas y el poco involucramiento masculino en el cuidado infantil.

Respecto a la situación más deseable para los varones, encontramos que un 9,6\% de los mismos opta porque el cuidado sea principalmente llevado a cabo por su pareja, lo que significa que, en este núcleo duro de varones, se expresa muy claramente la división sexual del trabajo, ya que ellos consideran como lo más deseable que durante la jornada laboral sean las mujeres las que 
se encarguen de sus hijos. Cabe señalar que ninguna mujer mencionó que lo más deseable era que el cuidado de niños pequeños fuera llevado a cabo por su pareja, lo cual indica la asociación por parte de las mujeres del cuidado como una acción exclusivamente femenina. En este mismo sentido, el 13,5\% de las mujeres optaría ser ellas principalmente las cuidadoras, lo cual es mencionado sólo por el 3,5\% de los varones.

Es importante destacar que una de cada cuatro personas optaría por compartir el cuidado con un centro infantil durante la jornada laboral, proporción no despreciable de la población en un contexto de reducidos y costosos servicios de atención para la población de estas edades en el país. Sobre este punto, cabe señalar que las mujeres se inclinan más que los varones a compartir el cuidado con centros infantiles (un 25,2\% frente a un 18,9\%), opinión que proviene justamente de quienes, en la práctica, dedican más tiempo al cuidado. Por tanto, si bien, para varones y mujeres, la situación más deseable es la atención domiciliaria y familiar, existe una mayor disposición femenina a exteriorizar los cuidados, lo que probablemente se explica porque son ellas las que efectivamente están enfrentadas a dicha situación.

En las hipótesis de investigación, establecíamos que el nivel socioeconómico era una variable que iba a influir en las representaciones sociales acerca del cuidado. Entre otras razones, porque aquello que la población afirma como situación más deseable está influenciado por la experiencia previa de modalidades de atención, relacionadas con las posibilidades de acceso a servicios.

Una de las líneas de investigación que puede considerarse una contribución para entender como las elecciones están basadas en la experiencia, es la desarrollada a partir del concepto de Elster de preferencias adaptativas (Pereira, 2007). Desde esta perspectiva, la población más vulnerable desarrolla preferencias adaptativas, las que surgen como una acomodación a situaciones de frustración por desear algo que no se puede alcanzar. La respuesta para eliminar esta frustración consiste en degradar lo deseado y otorgarle mayor valor a una alternativa que se encuentre dentro de lo realmente posible (Pereira, 2007).

Así, encontramos que existen variaciones en las situaciones más deseables de atención infantil según el nivel socioeconómico de los encuestados. Mientras que el $52,9 \%$ de los varones y el 58,3\% de las mujeres de nivel socioeconómico alto optarían porque el cuidado se resolviera en el marco familiar, entre los encuestados de nivel socioeconómico bajo esas cifras aumentan al 76,4\% y $61,6 \%$ para varones y mujeres, respectivamente.

Entendemos dicha diferencia en los distintos niveles socioeconómicos en función del marco de las preferencias adaptativas. Desde esta perspectiva, los niveles bajos desestiman las opciones de cuidado a las que no podrían acceder, desviando su elección por aquellas que son viables, como las opciones familiares.

Por otra parte, notamos que se registra mayor variación entre los varones según su nivel socioeconómico que entre las mujeres, lo que muestra que ellas tienden a mantener posiciones más homogéneas que ellos con mayor independencia del nivel socioeconómico. Así, encontramos que un tercio de 
Tabla 2. Edad en que cree que los niños y las niñas deben asistir a un centro infantil (porcentaje de la población total dividida por sexos)

\begin{tabular}{lccc}
\hline & Varones & Mujeres & Total \\
\hline Antes del año & 6,4 & 5,2 & 5,8 \\
Desde 1 año & 14,9 & 13,0 & 13,9 \\
Desde los 2 años & 18,6 & 28,4 & 23,8 \\
Desde los 3 años & 31,1 & 28,4 & 29,7 \\
Desde los 4 años o más & 27,4 & 24,3 & 25,8 \\
No sabe / No contesta & 1,6 & 0,7 & 1,1 \\
\hline Total & 100 & 100 & 100
\end{tabular}

Fuente: Encuesta Nacional sobre Representaciones Sociales del Cuidado. FCS-INMUJERES-ANII

los varones de nivel alto (un 32,7\%) se inclina por la modalidad de cuidado institucional. Por el contrario, los varones de nivel bajo son quienes muestran las representaciones sociales más familistas en torno al cuidado y solo un $12,1 \%$ de éstos se inclina por la atención institucional. Como muestran las encuestas de uso del tiempo realizadas en el país (Aguirre 2003b, INE, 2008), en la población perteneciente a los quintiles inferiores de ingresos se presentan las mayores brechas de género en el tiempo dedicado al trabajo no remunerado. Considerando este dato, podemos señalar que el familismo que observamos en este grupo de varones se traduce, en realidad, en mayor carga de trabajo femenino.

\subsection{El ingreso en el centro infantil, ¿cuál es la edad ideal?}

En este apartado, analizamos las opiniones de la población respecto a la edad en que se considera apropiado que los niños y las niñas comiencen a asistir a un centro infantil. Como se observa en la siguiente tabla, no hay consenso en la población sobre esta cuestión. Encontramos que el 43,5\% opina que deben concurrir antes de los 3 años; el 29,7\%, que deben concurrir a partir de esta edad, y el 25,8\% restante, que deben hacerlo luego de los 4 años. A pesar de que no se cuenta con una oferta universal de servicios de cuidado que cubra a niños y niñas menores de 3 años, queda evidenciado que un gran porcentaje de la población uruguaya considera que deben concurrir a un centro antes de esta edad.

Se observan diferencias entre varones y mujeres en este punto, ya que mientras el $28,4 \%$ de las mujeres considera que la mejor edad es desde los 2 años, solo el $18,6 \%$ de los varones cree lo mismo. Ellos se inclinan en mayor medida porque el ingreso de los niños en el centro infantil sea a partir de los 3 años (un $31,1 \%$ ). En este sentido, las mujeres están más dispuestas que los varones a enviar a los hijos al centro infantil a edades más tempranas. Nuevamente, queremos resaltar que son ellas quienes dedican más tiempo al cuidado de los críos, por lo que es más probable que la experiencia de cuidado esté influyendo más en ellas que en el caso de los varones. 
Otra hipótesis de trabajo indicaba que el contacto con situaciones de cuidado y la convivencia con la población dependiente también eran factores que influirían en las representaciones sociales acerca de la atención a los menores. De esta manera, comparamos las opiniones sobre la edad adecuada de asistencia de los niños al centro infantil correspondiente a la población en general y a la que pertenece a hogares en los que residen niños menores de 3 años. Encontramos que tanto varones como mujeres pertenecientes a hogares con menores de 3 años consideran que los niños deben concurrir a edades más tempranas a centros infantiles que la población en general. El 53,4\% de las personas de hogares con presencia de hijos menores de 3 años opina que la edad más adecuada es antes de los 3 años, mientras que esto ocurre con el 43,5\% de la población total. De todas formas, también sucede que, dentro de la población con niños menores de 3 años, los varones consideran que los críos deben asistir a los centros infantiles a edades más tardías que las mujeres pertenecientes a esa población.

Este apartado mostró que gran parte de la población optaría por resolver el cuidado de menores a través de centros infantiles de calidad para niños y niñas de 2 y 3 años. La oferta pública de servicios para esta población se concentra actualmente en los sectores con menos recursos, por lo que se aprecia una demanda insatisfecha respecto a dicha cuestión, lo cual indica la necesidad de apoyar a los hogares y a las familias para que puedan acceder al cuidado institucional de calidad para niños y niñas, así como para hacerlo compatible con el ejercicio de derechos de todos sus integrantes.

\section{3. ¿A qué edad es conveniente que los niños y niñas queden bajo el cuidado de otra persona en el hogar?}

Una de las modalidades frecuentes de cuidado que se dan actualmente en el marco de las soluciones privadas o brindadas por las familias es el cuidado en el hogar, provisto por una persona remunerada o no remunerada. En la siguiente tabla, observamos la edad en que la población considera adecuado dejar a los hijos al cuidado de otras personas durante la jornada laboral. El 55,2\% de los varones y el $50,0 \%$ de las mujeres creen que la mejor edad para ello es a partir de los 3 años. El 23,4\% de las personas considera que la edad ideal es antes de los 2 años. Si comparamos estos datos con los analizados sobre la edad ideal para enviar a los niños y niñas a un centro infantil, observamos que existe una menor disposición a dejar a hijos e hijas al cuidado de otras personas cuando son menores de 3 años, que para enviarlos a un centro infantil. Seguramente esto se deba a la falta de confianza que suele existir en torno a la contratación de una persona en el hogar que queda sola con los niños, mientras que existe mayor confianza en los centros infantiles, que cuentan con mayores controles. No existen en la actualidad mecanismos institucionales que vigilen la calidad del cuidado brindado en el domicilio, con lo que las familias tienen pocas herramientas para seleccionarlo y controlarlo. Este hecho probablemente explique la mayor disposición a brindar el cuidado a un centro infantil a edades tempranas. 
Tabla 3. Edad en que cree que los niños deberían quedar al cuidado de otra persona durante la jornada laboral (porcentaje de la población total dividida por sexos)

\begin{tabular}{lccc}
\hline & Varones & Mujeres & Total \\
\hline Entre 0 y 12 meses & 8,0 & 9,7 & 8,9 \\
Entre 12 y 24 meses & 16,4 & 12,8 & 14,5 \\
Luego de los 2 años & 11,7 & 19,7 & 15,9 \\
Luego de los 3 años & 55,2 & 50,0 & 52,4 \\
No sabe / No contesta & 8,8 & 7,8 & 8,3 \\
\hline Total & 100 & 100 & 100 \\
\hline
\end{tabular}

Fuente: Encuesta Nacional sobre Representaciones Sociales del Cuidado. FCS-INMUJERES-ANII

En los niveles socioeconómicos altos y medios, hay una mayor disposición a que los hijos se queden al cuidado de otras personas durante la jornada laboral a edades más tempranas que en los otros niveles.

Cuando comparamos la población en general con la población perteneciente a hogares con presencia de menores de 3 años, observamos que existe una tendencia de ésta última a adelantar la edad en que les parece adecuado dejarlos con otro adulto en el hogar durante la jornada laboral.

Por tanto, a las personas que tienen experiencia en el cuidado de los niños menores de 3 años por convivir con éstos, les parece adecuado dejarlos en el hogar a cargo de otra persona a edades más tempranas, así como recurrir a los servicios de un centro infantil durante la jornada laboral, como se mostró en el punto anterior.

\section{4. ¿Cuál es, para la población uruguaya, el «deber ser» sobre los roles de madres y padres en el cuidado de sus hijos e hijas?}

En el siguiente apartado, analizamos las opiniones de la población sobre las obligaciones respecto al cuidado infantil que tienen madres y padres en función de su «deber ser». Más de la mitad de la población considera que las madres están obligadas a cuidar personalmente de hijos e hijas menores de 1 año, mientras que solo una tercera parte cree que los padres tienen la misma obligación.

Por el contrario, con respecto a la frase sobre la obligación de garantizar el buen cuidado de los hijos e hijas, 6 de cada 10 personas creen que los padres varones están obligados a garantizarlo, mientras que tan sólo 4 de cada 10 personas cree lo mismo acerca de las madres.

Por lo tanto, hallamos que en el «deber ser» del cuidado predomina para las madres la obligación del cuidado directo, mientras que en el «deber ser» del cuidado de los padres predomina el garantizar el cuidado. Se evidencia nuevamente la vigencia de la división sexual del trabajo, que otorga a las mujeres el rol de cuidadoras y a los varones el de proveedores económicos. El garantizar el cuidado puede estar asociado a garantizar ciertas condiciones económicas, de infraestructura, en el funcionamiento de un hogar y, por lo tanto, es un 
Tabla 4. Opinión respecto al rol de las madres y de los padres en el cuidado de niños y niñas menores de 1 año (porcentaje de la población total dividida por sexos)

\begin{tabular}{|c|c|c|c|}
\hline & Varones & Mujeres & Total \\
\hline $\begin{array}{l}\text { Las madres están obligadas a cuidar personalmente } \\
\text { de sus hijos e hijas menores de } 1 \text { año }\end{array}$ & 54,0 & 56,6 & 55,4 \\
\hline $\begin{array}{l}\text { Las madres están obligadas a garantizar que sus } \\
\text { hijos e hijas menores estén bien atendidos, pero } \\
\text { no es imprescindible que participen directamente } \\
\text { en el cuidado }\end{array}$ & 42,1 & 40,3 & 41,1 \\
\hline No sabe / No contesta & 4,0 & 3,1 & 3,5 \\
\hline \multirow[t]{2}{*}{ Total } & 100 & 100 & 100 \\
\hline & Varones & Mujeres & Total \\
\hline $\begin{array}{l}\text { Los padres varones están obligados a cuidar } \\
\text { personalmente de sus hijos e hijas menores de } 1 \text { año }\end{array}$ & 35,2 & 33,9 & 34,5 \\
\hline $\begin{array}{l}\text { Los padres varones están obligados a garantizar } \\
\text { que sus hijos e hijas menores de } 1 \text { año estén bien } \\
\text { atendidos, pero no es imprescindible que participen } \\
\text { directamente en el cuidado }\end{array}$ & 61,6 & 62,1 & 61,9 \\
\hline No sabe / No contesta & 3,2 & 4,0 & 3,6 \\
\hline Total & 100 & 100 & 100 \\
\hline
\end{tabular}

Fuente: Encuesta Nacional sobre Representaciones Sociales del Cuidado. FCS-INMUJERES-ANII

rol que puede resultar más fácilmente asimilable a los padres varones. Por el contrario, el rol de cuidado directo, del vínculo íntimo con los niños menores de 1 año, es asociado a las madres.

Este «deber ser» del cuidado impacta sobre la dedicación de tiempo de unos y otras a esta tarea y hace que las mujeres dediquen más horas de cuidado directo, mientras que ellos incrementan sus horas de trabajo remunerado en el mercado cuando tienen hijos, tal como lo han evidenciado las encuestas de uso del tiempo y las encuestas de hogares. Así pues, la tasa de actividad es de un $68,9 \%$ para las mujeres de 14 a 49 años que no tienen niños menores de 4 años en el hogar y de un $35,7 \%$ con tres o más menores. Por el contrario, los varones aumentan la tasa de actividad, que va de un $80,6 \%$ cuando no tienen hijos a un $90,0 \%$ con tres o más niños (Sistema de Información de GéneroInmujeres, 2012).

Cuando consideramos el nivel socioeconómico, las opiniones son similares entre personas del mismo estrato de forma independiente de su sexo. Para los estratos medios, el porcentaje de quienes creen que las mujeres están obligadas a ejercer el cuidado directo es menor que para el promedio de la población, mientras que en los estratos más bajos es mayor que el promedio. Las personas de niveles socioeconómicos más altos están más de acuerdo con la obligación de cuidado indirecto que con la de cuidado directo que las personas de niveles bajos, tanto respecto a las madres como a los padres. Se observa como la división sexual del trabajo manifestada en las representaciones es más rígida en los niveles socioeconómicos inferiores. 
Tabla 5. Porcentaje de varones y mujeres según qué acciones emprendería si tuviera hoy un hijo menor de 1 año y en su empleo no le permitieran tomarse licencia adicional (porcentaje de población ocupada)

\begin{tabular}{|c|c|c|c|c|c|}
\hline & \multicolumn{3}{|c|}{ Población ocupada total } & \multicolumn{2}{|c|}{$\begin{array}{l}\text { Población ocupada con } \\
\text { presencia de menores } \\
\text { de } 1 \text { año }\end{array}$} \\
\hline & Varones & Mujeres & Total & Varones & Mujeres \\
\hline $\begin{array}{l}\text { Mantendría su empleo con } \\
\text { el horario actual }\end{array}$ & 29,3 & 20,6 & 25,5 & 23,5 & 38,5 \\
\hline $\begin{array}{l}\text { Seguiría con el empleo, aunque } \\
\text { reduciría horario e ingresos }\end{array}$ & 52,2 & 41,7 & 47,5 & 58,8 & 38,5 \\
\hline $\begin{array}{l}\text { Abandonaría provisoriamente } \\
\text { el empleo para dedicarse al } \\
\text { cuidado del niño }\end{array}$ & 10,1 & 21,5 & 15,2 & 17,6 & 15,4 \\
\hline $\begin{array}{l}\text { Abandonaría definitivamente el } \\
\text { empleo para cuidar al niño }\end{array}$ & 5,1 & 12,6 & 8,4 & 0,0 & 7,7 \\
\hline No opina & 2,9 & 3,6 & 3,2 & 0,0 & 3,1 \\
\hline Total & 100 & 100 & 100 & 100 & 100 \\
\hline
\end{tabular}

Fuente: Encuesta Nacional sobre Representaciones Sociales del Cuidado. FCS-INMUJERES-ANII

\subsection{Para afrontar el cuidado infantil, ¿qué decisiones toman varones y mujeres} luego de su licencia maternal o paternal?

En este apartado, analizamos los diferentes comportamientos de varones y mujeres en relación con su participación en el mercado laboral cuando se ven enfrentados a las demandas de cuidado infantil. Frente a un contexto legal que ofrece licencias muy reducidas ${ }^{7}$ para el cuidado de niños y niñas pequeños en Uruguay, se vuelve relevante analizar las decisiones que varones y mujeres trabajadores toman ante las rígidas disposiciones del mercado laboral.

Frente a la imposibilidad de conseguir licencia adicional luego de la licencia maternal o paternal, el $34,1 \%$ de las mujeres ocupadas estarían dispuestas a abandonar su empleo en caso de tener que cuidar a un hijo o a una hija menor de 1 año. Sin embargo, sólo el 15,2\% de los varones ocupados tomarían esta decisión.

En los hogares donde están enfrentadas a la situación real de necesidades de cuidado infantil, es decir, donde hay presencia de niños menores de 1 año, las mujeres presentan menor disposición a abandonar el empleo, lo que no ocurre en el caso de los varones. Por tanto, aquellas mujeres que se encuentran en la situación de cuidado de niños menores de 1 año están menos dispuestas a abandonar su trabajo que las de la población en general, por lo que las políticas

7. En el año 2013, se aprueba la Ley 19.161, que amplía y equipara la extensión de la licencia maternal de las mujeres del sector privado con las del público, con una duración de 14 semanas, mínimo exigido por las recomendaciones internacionales (OIT, Convenio sobre la protección de la maternidad, 2000) y fija en 13 días la licencia para los varones. 
de cuidado deberían apuntar a que estas mujeres puedan sostener el empleo al mismo tiempo que garantizar el cuidado de calidad para sus hijos.

\section{Principales conclusiones}

Una primera constatación es la fuerte presencia del familismo en las representaciones sociales del cuidado infantil de la población uruguaya. Para la mayor parte de dicha población, la situación más deseable para atender a los niños pequeños es la que se brinda en domicilio y, especialmente, por medio de los miembros de las familias. Este familismo tan marcado en las opiniones de la población ubica a las familias como el actor con mayor capacidad y legitimidad para brindar cuidado de calidad a los niños pequeños y representa un desafío a considerar en el diseño de políticas que ofrezcan servicios de cuidado institucionales para criaturas en edades tempranas. La inexistencia o la mínima presencia de servicios estatales dirigidos a niños y a niñas más pequeños puede estar influyendo en la elección familiar tan marcada en la población uruguaya.

En segundo lugar, se constata una relación directa entre el familismo y el nivel socioeconómico: a menor nivel socioeconómico, se observa mayor familismo en las situaciones consideradas ideales para el cuidado. Esto se visualiza también en las edades ideales de inicio del cuidado institucional o extrafamiliar, de modo que las personas de niveles socioeconómicos más altos se inclinan a dejar a sus hijos al cuidado de personas contratadas o de instituciones a edades más tempranas.

Esta relación está probablemente vinculada al acceso desigual a modalidades de cuidado institucional de calidad, dado que actualmente, en Uruguay, es muy baja la cobertura de centros de cuidado de calidad para niños menores de 3 años, y la misma está segmentada por niveles socioeconómicos ${ }^{8}$. Esto implica que, entre las personas con más alto nivel de ingresos, la presencia de instituciones de atención infantil es más frecuente y, al tratarse de una experiencia conocida, incide en su elección como opción de cuidado. Por el contrario, para la población perteneciente a niveles socioeconómicos más bajos, la experiencia cercana es la del cuidado familiar, debido a la escasez de recursos económicos para costear servicios de calidad.

Se advierte, entonces, que las representaciones sociales de la asistencia a menores están mediadas por el nivel socioeconómico y se vinculan con las experiencias de cuidado vividas o conocidas, tal como se esperaba en las hipótesis de partida. Esto puede explicarse a partir del marco interpretativo de las preferencias adaptativas mencionado anteriormente. En este sentido, el Sistema Nacional de Cuidados, mediante la oferta de nuevos servicios, deberá generar una distribución más equitativa de las atenciones institucionales de calidad que transforme las representaciones actuales, a fin de reducir la carga de trabajo de cuidados realizado por las familias.

8. Para más detalles, consultar Salvador (2011), Hacia un sistema nacional de cuidados en el Uruguay [en línea], <http://www.eclac.org/publicaciones/xml/2/44532/SerieSyC-Cuidados-Uruguay.pdf $>$. 
Ahora bien, las diferencias señaladas son más relevantes en el caso de los varones. Entre las mujeres, las respuestas varían mucho menos en función de las variables estudiadas. Efectivamente, y en tercer lugar, se constata la fuerza del mandato de género respecto al rol cuidador en la identidad femenina, que trasciende las distintas posiciones sociales: si bien las mujeres no constituyen un grupo homogéneo, muestran mayor similitud como colectivo que los varones, puesto que comparten representaciones similares. De esta forma, como estaba planteado en las hipótesis iniciales, encontramos que la variable sexo tiene un peso significativo en las representaciones sociales del cuidado infantil.

En esta misma línea, se observa la persistencia de la división sexual del trabajo en relación con el «deber ser» del cuidado: por un lado, en el «deber ser» que la población atribuye a los varones, hay una tendencia a percibirlos como los responsables de garantizar el cuidado, aludiendo a su rol de proveedores económicos; por otro lado, el «deber ser» de las mujeres las asocia a su rol de cuidadoras directas, lo cual implica un vínculo íntimo. Por tanto, es fundamental que el Sistema de Cuidados promueva cambios culturales que transformen el «deber ser» de mujeres y varones respecto al rol cuidador hacia uno de responsabilidad compartida.

En cuarto lugar, se evidencia la tendencia de las mujeres a flexibilizar su situación en el mercado laboral en función de las necesidades de atención infantil. Se observa aquí de qué modo el "deber ser» atribuido a las mujeres impacta en su inserción y permanencia en el mercado de empleo. Con el fin de garantizar el derecho al trabajo de las mujeres y la igualdad de oportunidades, se hace necesario que el mercado laboral incorpore a la reproducción social como factor indispensable para la producción y que se implementen políticas de empleo que garanticen el derecho a cuidar y a ser cuidado.

Finalmente, los hallazgos de investigación presentados en este artículo constituyen un aporte valioso para que el Sistema de Cuidados conozca las barreras culturales a las que se enfrenta al intervenir en la distribución de la asistencia a menores, a fin de generar acciones que no reproduzcan la división sexual del trabajo presente en las representaciones orientadoras de las prácticas y que permitan asumir la atención a las criaturas como una responsabilidad social.

\section{Referencias bibliográficas}

Aguirre, R. (2003a). Género, ciudadanía social y trabajo. Montevideo: DS-FCSUDELAR. Doble Clic.

- (2003b). Trabajo no remunerado y uso del tiempo. Fundamentos conceptuales y avances empiricos. La encuesta Montevideo 2003. El Uruguay desde la Sociología II. Montevideo: DS-FCS-UDELAR.

- (2008). «El futuro del cuidado». En: Arriagada, I. (ed.). Futuro de las familias y desafios para las politicas. Santiago de Chile: CEPAL / ONUSIDA / UNIFEM / UNFPA.

Aguirre, R. (ed.) (2009). Las bases invisibles del bienestar social: El trabajo no remunerado en Uruguay. Montevideo: Fondo de Desarrollo de las Naciones Unidas para la Mujer (UNIFEM). 
Aguirre, R. y BAtThyÁnY, K. (2005). Uso del tiempo y trabajo no remunerado: Encuesta en Montevideo y área metropolitana 2003. Montevideo: Universidad de la República. UNIFEM.

Araya Umaña, S. (2002). Las representaciones sociales: Ejes teóricos para su discusión. Costa Rica: FLACSO. (Cuaderno de Ciencias Sociales; 127)

ArriagadA, I. (2007). «Transformaciones familiares y políticas de bienestar en América Latina». En: Arriagada, I. (coord.). Familias y políticas públicas en América Latina: Una historia de desencuentros. Santiago de Chile: CEPAL.

BATTHYÁnY, Karina (200I). Respuestas institucionales a las necesidades de cuidado infantil en el Uruguay: Diagnóstico de situación. Montevideo: DS-FCS-UDELAR.

- (2002). Maternidad y trabajo asalariado. Las estrategias de cuidado infantil de las mujeres en Montevideo. Estudio de caso múltiple. El Uruguay desde la Sociología I. Montevideo: DS-FCS-UDELAR.

- (2004). Cuidado infantil y trabajo: ¿un desafío exclusivamente femenino?: Una mirada desde el género y la ciudadanía social. CINTERFOR/OIT.

- (2009). "Cuidado de personas dependientes y género». En: Aguirre, R. (ed.). Las bases invisibles del bienestar social: El trabajo no remunerado en Uruguay. Montevideo: Fondo de Desarrollo de las Naciones Unidas para la Mujer (UNIFEM).

- (2010). «Trabajo no remunerado y división sexual del trabajo: Cambios y permanencias en las familias». En: El Uruguay desde la Sociología VIII. Montevideo: DS-FCS-UDELAR.

Carrasco, C.; Borderías, C. y Torns, T. (2011). «El trabajo de cuidados: Antecedentes históricos y debates actuales». En: Carrasco, C.; Borderías, C. y Tonns, T. (eds.). El trabajo de cuidados: Historia, teoria y politicas. Madrid: Catarata.

Consejo Nacional de Política Social (2012). Hacia un modelo solidario de cuidados: Propuesta para la construcción de un Sistema Nacional de Cuidados. Aprobado por el Gabinete Social 20 de setiembre. [Documento del grupo de trabajo]

Daly, M. y Lewis, J. (2011). «El concepto de social care y el análisis de los estados de bienestar contemporáneos». En: Carrasco, C.; Borderías, C. y Torns, T. (eds.). El trabajo de cuidados: Historia, teoría y políticas. Madrid: Catarata.

Durán, M. A. (2003). El trabajo no remunerado y las familias: Consulta técnica sobre contabilización de la producción no remunerada de servicios de salud en el hogar. Washington D. C.: Unidad de Género y Salud y Unidad de Políticas y Sistemas de la Organización Panamericana de la Salud.

Esping-Andersen, G. (2000). Fundamentos sociales de las economias postindustriales. Madrid: Ariel.

Hochschild, R. (2003). La mercantilización de la vida intima: Apuntes de la casa y el trabajo. Madrid: Katz.

Instituto Nacional de Estadística (INE) (2008). Uso del tiempo y trabajo no remunerado: informe sobre el Módulo de la Encuesta Continua de Hogares. Montevideo: UNIFEM/INE/INMUJERES/UDELAR. Disponible en: www.ine.gub.uy.

Letablier, M. Th. (2001). "Le travail centré sur autrui et sa conceptualisation en Europe». Travail, Genre et Societés [en línea], 6, París: L'Harmattan. <http://dx.doi.org/10.3917/tgs.006.0019>.

Martínez Franzoni, J. (2009). Domesticar la incertidumbre en América Latina: Mercado laboral, política social y familias. San José (Costa Rica): Editorial de la Universidad de Costa Rica / UNDP. 
Martínez Franzoni, J. y Voorend, K. (2013). "Desigualdades de género en los regímenes de bienestar latinoamericanos: Mercado, política social y organización familiar de los cuidados». En: Pautassi, Laura y Ziebecchi, Carla. Las fronteras del cuidado: Agenda, derechos e infraestructura. Buenos Aires: Biblos.

Montaño, Sonia (2010) «El Cuidado en Acción, entre el derecho y el trabajo». En: Montaño, Sonia y Calderón, Coral (coordinadores). El Cuidado en Acción. Santiago de Chile: Cuadernos de la CEPAL, 94.

Moscovici, S. (1979). El psicoanálisis, su imagen y su público. Buenos Aires: Huemul.

Navarro, Vicenç (2005). «Bienestar insuficiente, democracia incompleta». Ponencia presentada en la VI Escuela Internacional de Verano UGT Asturias. Avilés, del 12 al 16 de abril de 2005. Reinventando el Estado de Bienestar: ¿Nuevas políticas para iguales objetivos?

PAutassi, Laura (2010). "Cuidado y derechos: La nueva cuestión social». En: MonTAÑo, S. y Calderón, C. (cords.). El cuidado en acción: Entre el derecho y el trabajo. Santiago de Chile: Cepal.

Pereira, Gustavo (2007). «Preferencias adaptativas: Un desafío para el diseño de las políticas sociales». Revista de Filosofía Moral y Política [en línea], 36, enero-junio, 143-165, ISEGORÍA. <http://dx.doi.org/10.3989/isegoria.2007.i36.63>.

Pérez Orozco, A. (2006). Perspectivas feministas en torno a la economía: El caso de los cuidados. Madrid: Consejo Económico y Social. (Estudios; 190)

Sarraceno, Chiara (1994) "A dependencia construida e a independencia negada: estruturas de género da cidadania». En: Bonacchi, G. y Groppi, A. (orgs). O dilema da cidadania. San Pablo: Unesp

SAInsbury, Diane (2000) «Les droits sociaux des femmes et des hommes. Les dimensions de genre dans les états providence». En: Revista Genre et politique. Debats et perspectives. Folio Essais Gallimard: France.

Sistema de Información DE GÉNero (SIG) (2012). Estadisticas de Género, 2011 [en línea]. Montevideo: Inmujeres / MIDES / UNFPA.

<http://www.inmujeres.gub.uy/innovaportal/file/20979/1/estadisticas_de_genero_2011_final.pdf>. 